\title{
Passive isolator design for jitter reduction in the Terrestrial Planet Finder Coronagraph
}

\author{
Carl Blaurock ${ }^{a}, K_{\text {Kuo-Chia Liu }}{ }^{b}$, Larry Dewell ${ }^{c}$, James Alexander ${ }^{d}$ \\ ${ }^{2}$ Nightsky Systems, Inc. 3916 Lauriston Rd., Raleigh NC 27616 \\ bNASA Goddard Space Flight Center, 8800 Greenbelt Road, Greenbelt, MD 20771 \\ 'Lockheed Martin Space Systems, San Jose, CA 94304 \\ dNASA Jet Propulsion Laboratory, Palo Alto, CA 91109
}

\begin{abstract}
Terrestrial Planet Finder (TPF) is a mission to locate and study extrasolar Earthlike planets. The TPF Coronagraph (TPF-C), planned for launch in the latter half of the next decade, will use a coronagraphic mask and other optics to suppress the light of the nearby star in order to collect visible light from such planets. The required contrast ratio of 5e11 can only be achieved by maintaining pointing accuracy to 4 milli-arcseconds, and limiting optics jitter to below 5 $\mathrm{nm}$. Numerous mechanical disturbances act to induce jitter. This paper concentrates on passive isolation techniques to minimize the optical degradation introduced by disturbance sources. A passive isolation system, using compliant mounts placed at an energy bottleneck to reduce energy transmission above a certain frequency, is a low risk, flight proven design approach. However, the attenuation is limited, compared to an active system, so the feasibility of the design must be demonstrated by analysis. The paper presents the jitter analysis for the baseline TPF design, using a passive isolation system. The analysis model representing the dynamics of the spacecraft and telescope is described, with emphasis on passive isolator modeling. Pointing and deformation metrics, consistent with the TPF-C error budget, are derived. Jitter prediction methodology and results are presented. Then an analysis of the critical design parameters that drive the TPFC jitter response is performed.
\end{abstract}

Keywords: $\quad$ Coronagraph, jitter, reaction wheels, attitude control system, pointing control system, image control.

\section{INTRODUCTION}

The Terrestrial Planet Finder (TPF) is one of the proposed missions under NASA's Origins Program, aimed to search for habitable extra-solar planets and life beyond Earth. Currently there are two promising architectures viable for extrasolar planet detection: visible-light coronagraph, and infrared interferometer. The studies described in this paper focus on the Coronagraph architecture that meets the minimum science requirement ${ }^{1}$. For this mission, the system must suppress the bright light generated from the planet's star, allowing the fainter, reflected light from the planet to be observed. To provide the requisite contrast ratio, the dynamic stability of the system must be maintained in the submilliarcsecond/sub-micrometer level, despite the presence of structural vibration and perturbations caused by internal mechanisms and external disturbances.

The design effort is currently carrying two design options for suppression of induced jitter: passive isolation and active isolation. Both concepts are designed to limit energy transmission from a dynamically noisy host structure (the support module) to a quiet payload carrying all of the optics. The passive isolation design uses soft flexures to reduce the transmission of energy from the disturbances to the optics. The active design actively decouples the payload from the disturbances. In this paper, we focus on the Flight Baseline Cycle 1 (FB1) passively isolated pointing control system (PCS) for TPF Coronagraph. The goals of the analysis are to compare the predicted jitter with allocated jitter; to hand off RMS optical jitter to the optical modeling team for contrast simulation; and to generate engineering requirements for actuator stroke, bandwidth, and resolution, control system sample rates and gains, and sensor noise and bandwidth.

The paper first presents the requirements on jitter suppression, and outlines the passive isolation design. Once the PCS is defined, an integrated model can be formed by incorporating dynamic models from various disciplines, including structures, optics, and controls. Such a model allows us to perform end-to-end analyses and to predict the behavior of rigid body motion, image motion, and wave front error (WFE) from different disturbance sources. The focus of this paper is on flowing the disturbances through a frequency domain model of the system to structural, optical, and contrast responses.

In Section 2, the overall PCS design and related engineering requirements are presented. Following the general description of the system, a brief summary of the component models used in the integrated model is given in Section 3. 
After obtaining a suitable integrated model, the attention turns to evaluating the performance of the integrated system. The equations that flow optical responses to contrast are summarized in Section 4 and the results from the linear model jitter analysis is presented in Section 5. Finally, summary of our analysis results and future work are provided in Section 6.

\section{POINTING CONTROL SYSTEM DESIGN}

The following subsections present the top level PCS requirements and the FB1 passive isolation design.

\subsection{Requirements}

The top level jitter requirements are formulated in terms of contrast $^{6}$. The contrast has contributions from the following sources:

- Line of sight (centration) error: motion of the centroid on the occulting mask. The FBI model uses a linear mask aligned with the long axis of the Primary Mirror, so that only the centroid motion in the $\mathrm{X}$ axis influences the contrast.

- Beam walk: motion of the chief ray along each of the optical elements.

- Aberrations: distortion of the wave front at the mask, caused by relative motion of the optical elements ("structural deformation") and bending of the optical elements ("optical deformation"). For the Flight Baseline Cycle 1, only deformation of the Primary is considered.

- Mask error: due to wave front error at the mask.

The error budget defines requirements on physical motion of the telescope in terms of rigid body pointing (RBP) of the telescope, 6-DOF rigid-optics motion of the 21 optical elements, and deformation of the first 4 mirrors in terms of RMS-normalized Zernike amplitudes. Physical motions are then flowed to optical metrics. The optical metrics are then flowed to contrast contributions. The error budget assumes that optical motions are independent. The requirements on each of the contrast sources are given in Table 2-1. Contrast is defined as the ratio of light intensities, so is unitless.

\subsection{TPF-C system overview}

The TPF-C Flight Baseline 1 (FB1) design consists of an optical payload supported by a host spacecraft termed the "support module" (Figure 2-1). The support module provides power, communications, coarse attitude control, and passive thermal control. Dynamically significant components of the support module entail solar arrays, antennas, reaction wheels and a solar sail, and a sunshield, respectively. The payload encompasses an optics train consisting, in the FB1 design, of 21 optics running from an $8 \mathrm{~m}$ by $3.5 \mathrm{~m}$ elliptical primary, to a Secondary Mirror (SM) mounted at the tip of a $12 \mathrm{~m}$ tower, through the remaining optics to the occulting mask. The payload and support module are connected by cables for power and data, as well as the isolation system (either passive or active). This paper will concentrate on the passive design alternative.

Table 2-1 Contrast requirement by contribution.

\begin{tabular}{|l|l|}
\hline Contribution & Requirement \\
\hline Beam walk & $1.9 \mathrm{e}-012$ \\
\hline LOS jitter & $9.0 \mathrm{e}-014$ \\
\hline LOS mask error & $5.5 \mathrm{e}-013$ \\
\hline Structural Deformation aber. & $2.8 \mathrm{e}-017$ \\
\hline SD aber. maskerror & $1.7 \mathrm{e}-017$ \\
\hline Optical deformation aber. & $8.5 \mathrm{e}-013$ \\
\hline OD aber. maskerror & $5.2 \mathrm{e}-015$ \\
\hline Total & $3.4 \mathrm{e}-012$ \\
\hline
\end{tabular}




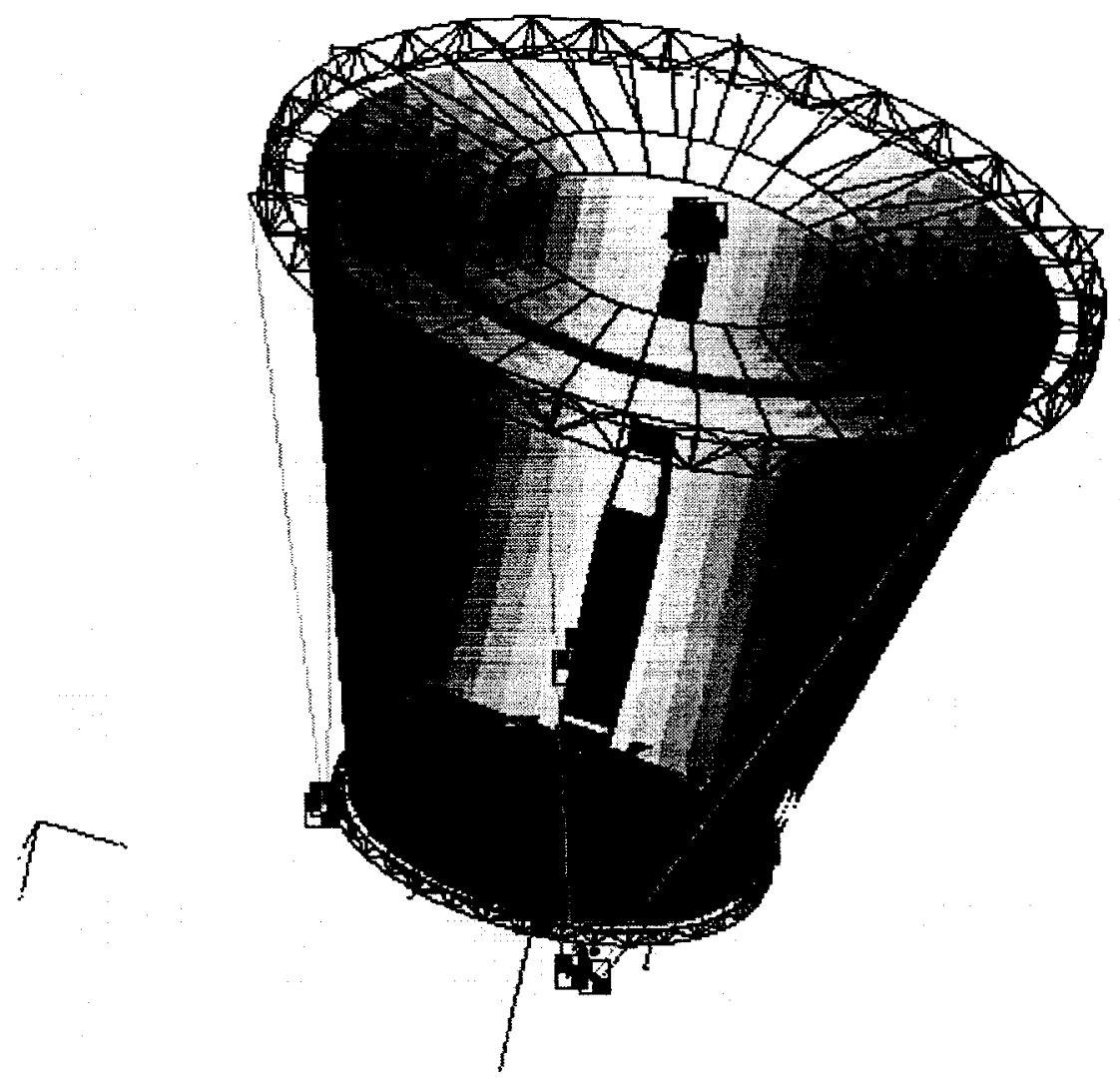

Figure 2-1 TPF-C Flight Baseline 1 design. Solar sail and solar array not shown.

\subsection{Staged pointing control}

The PCS must maintain the physical motions of the optical elements, and the position of the centroid on the mask, to within the requirements. The design approach is to locate intrinsically noisy components, such as reaction wheels, on a noisy platform, and then limit the energy transmission into the optical payload. An image control system is then used to actively control residual pointing error.

\subsection{Passive isolation}

Passive isolation is used to attenuate disturbance energy transmission from the reaction wheels to the telescope. The passive isolation design effort focused on the need to apply realistic constraints to the performance of a passive isolator, while not introducing excessive conservatism. The approach was to identify the limits to passive isolator performance, model each effect (or demonstrate that the effect could be designed around), and tie the design to published specifications of existing designs.

\subsubsection{Limits to passive isolator performance}

The general response of a passive isolator is that of a low-pass filter. Simple models of passive isolators show a $40 \mathrm{~dB} /$ decade attenuation above the break frequency. Physical isolators exhibit an "isolation floor" where the transmissibility rolls from $40 \mathrm{~dB} /$ decade to (approximately) flat. There are four effects that drive isolator performance away from the $40 \mathrm{~dB} / \mathrm{dec}$ ade attenuation of the simplified isolator model:

- "lockup" of the isolator damper: a pure damper acts as a rate feedback that transmits force with a $+20 \mathrm{~dB} / \mathrm{dec}$ de gain compared to a pure spring.

- non-ideal response of the payload and spacecraft structures: the simple damper model applies when the payload and structure roll off at $40 \mathrm{~dB} / \mathrm{decade}$. A structure will roll off between 10 and $30 \mathrm{~dB} / \mathrm{decade}$ above its 
first mode. If the payload and spacecraft roll off at an average $20 \mathrm{~dB} / \mathrm{decade}$ each, the transmissibility from disturbances, through both structures and the isolator, to the sensitive location, will be flat.

- geometry: the hexapod arrangement of a typical isolator leads to coupling between the isolators that leads to a floor at high frequency

- cross-axis modes of the isolator: these modes can couple into the axial response, leading to localized high frequency amplification.

The TPF-C passive isolation design deals with these effects in the following manner. Damper lockup can be controlled by introducing a spring in series with the damper, that effectively filters high frequency energy transmission. The latter three effects are captured in the TPF-C analysis through the use of a physical isolator model that couples the payload and spacecraft, and that includes the cross-axial modes of the isolator.

The TPF-C isolator stages are placed between the Reaction Wheel Assembly (RWA) and the spacecraft, and between the payload and the spacecraft. An alternative design would place individual isolators on each reaction wheel, in place of the RWA isolator. The advantages of the RWA isolator are that it increases the suspended mass, enabling a lower break frequency, and that it requires fewer isolators, which should lower the cost.

\subsubsection{Reaction wheel assembly isolation}

The RWA isolator is a hexapod design using struts based on Honeywell D-struts. The RWA mass is $61.4 \mathrm{~kg}$, and the target frequency is $1.5 \mathrm{~Hz}$. Each strut is modeled in NASTRAN with 3 nodes, two CBAR elements, two CONM2 elements, and 12 CELAS2 lumped stiffnesses. A 4,2000 N/m flexure is assumed. The strut has a first bending (crossaxis) mode of $102 \mathrm{~Hz}$.

\subsubsection{Payload isolation}

For the FB1 design, the payload isolator is modeled as a set of 3-axis springs at each of the spacecraft/support module attachment locations. The springs are $30,000 \mathrm{~N} / \mathrm{m}$ each.

\subsection{Image stabilization}

The image stabilization system uses several nested active control loops to remove residual LOS error, due to disturbances that propagate through the isolators.

\subsubsection{Active control architecture}

The active control system uses a Fine Guidance Sensor (FGS) that senses light reflected from the mask to measure centroid position. Since most of the light from the target star is reflected, the FGS readout rate can be relatively high, between 100 and $500 \mathrm{~Hz}$. The FB1 design uses the Secondary Mirror, in addition to the Steering Mirror, as a LOS control actuator for the following reason. The $8^{\text {th }}$ order mask significantly reduces sensitivity to wavefront error, so that the largest contrast contributor is beam walk along the optics. Beam walk is defined by the chief ray, which by definition goes through the center of the Deformable Mirror. If the Secondary Mirror is then used to center the spot on the occulting mask in the presence of a rigid body pointing angle, the chief ray will have zero beam walk on all optics downstream of the SM. Since the bandwidth of the SM is limited by the structural response of the tower, a Fine Guidance Mirror is required to control high frequency LOS errors. The FGM is small and light enough that it does not interact significantly with structural modes, and therefore the bandwidth can approach the sample-rateconstrained limit of 10-50 Hz. The SM and FGM only control relative motion within the telescope, so reaction wheels are required to reject external disturbance

Table 2-2 Control system parameters.

\begin{tabular}{|c|c|c|c|c|c|}
\hline $\begin{array}{l}\text { Control } \\
\text { loop }\end{array}$ & Parameter & Value & $\begin{array}{l}\text { Sample } \\
\text { Freq. }\end{array}$ & Margins & Bandwidth \\
\hline$\overline{\text { FGM }}$ & $\begin{array}{l}\text { Break freq. } \\
\text { Lead }\end{array}$ & $\begin{array}{l}1 \mathrm{~Hz} \\
45^{\circ}\end{array}$ & $500 \mathrm{~Hz}$ & $\begin{array}{l}7.01 \mathrm{~dB} \\
25.6^{\circ} \\
\end{array}$ & $25.1 \mathrm{~Hz}$ \\
\hline SM & $\begin{array}{l}\text { Break freq. } \\
\text { Lead }\end{array}$ & $\begin{array}{l}0.001 \mathrm{~Hz} \\
45^{\circ}\end{array}$ & $\begin{array}{l}1000 \\
\mathrm{~Hz}\end{array}$ & $\begin{array}{l}49.11 \mathrm{~dB} \\
45.7^{\circ}\end{array}$ & $0.1 \mathrm{~Hz}$ \\
\hline ACS & $\begin{array}{l}\text { Crossover } \\
\text { Integral T.C. ratio } \\
\text { Estimator freq. } \\
\text { Elliptical order } \\
\text { Elliptical ripple } \\
\text { Elliptical atten. } \\
\text { Elliptical freq. }\end{array}$ & $\begin{array}{l}0.016 \mathrm{~Hz} \\
0.075 \\
10 \mathrm{~Hz} \\
3 \\
1 \mathrm{~dB} \\
30 \mathrm{~dB} \\
0.56 \mathrm{~Hz}\end{array}$ & $2 \mathrm{~Hz}$ & $\begin{array}{l}9.3 \mathrm{~dB} \\
34.8^{\circ}\end{array}$ & $0.043 \mathrm{~Hz}$ \\
\hline $\begin{array}{l}\text { RW speed } \\
\text { control }\end{array}$ & $\begin{array}{l}\text { Bandwidth } \\
\text { Lead }\end{array}$ & $\begin{array}{l}1 \mathrm{~Hz} \\
60^{\circ}\end{array}$ & $100 \mathrm{~Hz}$ & & $1 \mathrm{~Hz}$ \\
\hline
\end{tabular}


torques and offload the FGM and SM at low frequency.

\subsubsection{Control modes}

The PCS must operate in several modes during acquisition and imaging. In the coarse (slewing) mode, when the telescope is retargeted to a new star, the attitude sensors consist of Star Trackers for attitude and gyros for rate. In the acquisition mode, the telescope uses Payload Star Acquisition Cameras to center the star in the FGS field of view. In the fine (imaging) mode, the staged image stabilization control system described above is operational.

\subsubsection{Fine Guidance Mirror control}

The FGM control consists of a second order low pass filter with a lead compensator. The bandwidth is $25 \mathrm{~Hz}$ for the FB1 design, in order to control LOS errors introduced by a pair of $10 \mathrm{~Hz}$ modes. The filter break frequency is $1 \mathrm{~Hz}$, and the damping ratio is 0.707 .

\subsubsection{Secondary Mirror control}

The SM controller also consists of a second order low pass filter with lead. The break frequency was set to $0.001 \mathrm{~Hz}$ in order to suppress thermally induced SM-PM relative motion. The bandwidth was initially set to $1 \mathrm{~Hz}$ to offload the FGM, however the laser truss sensor noise (which was amplified by the SM control loop in the 1-5 $\mathrm{Hz}$ range) excited the isolator modes in the $1-4 \mathrm{~Hz}$ range. The SM bandwidth was dropped to $0.1 \mathrm{~Hz}$.

\subsubsection{Attitude control}

The attitude control system is a Proportional-Integral-Derivative (PID) design that was developed for the Next Generation Space Telescope Yardstick design ${ }^{2}$. It uses structural filters to suppress the first flexible modes of the structure. The bandwidth is $0.016 \mathrm{~Hz}$.

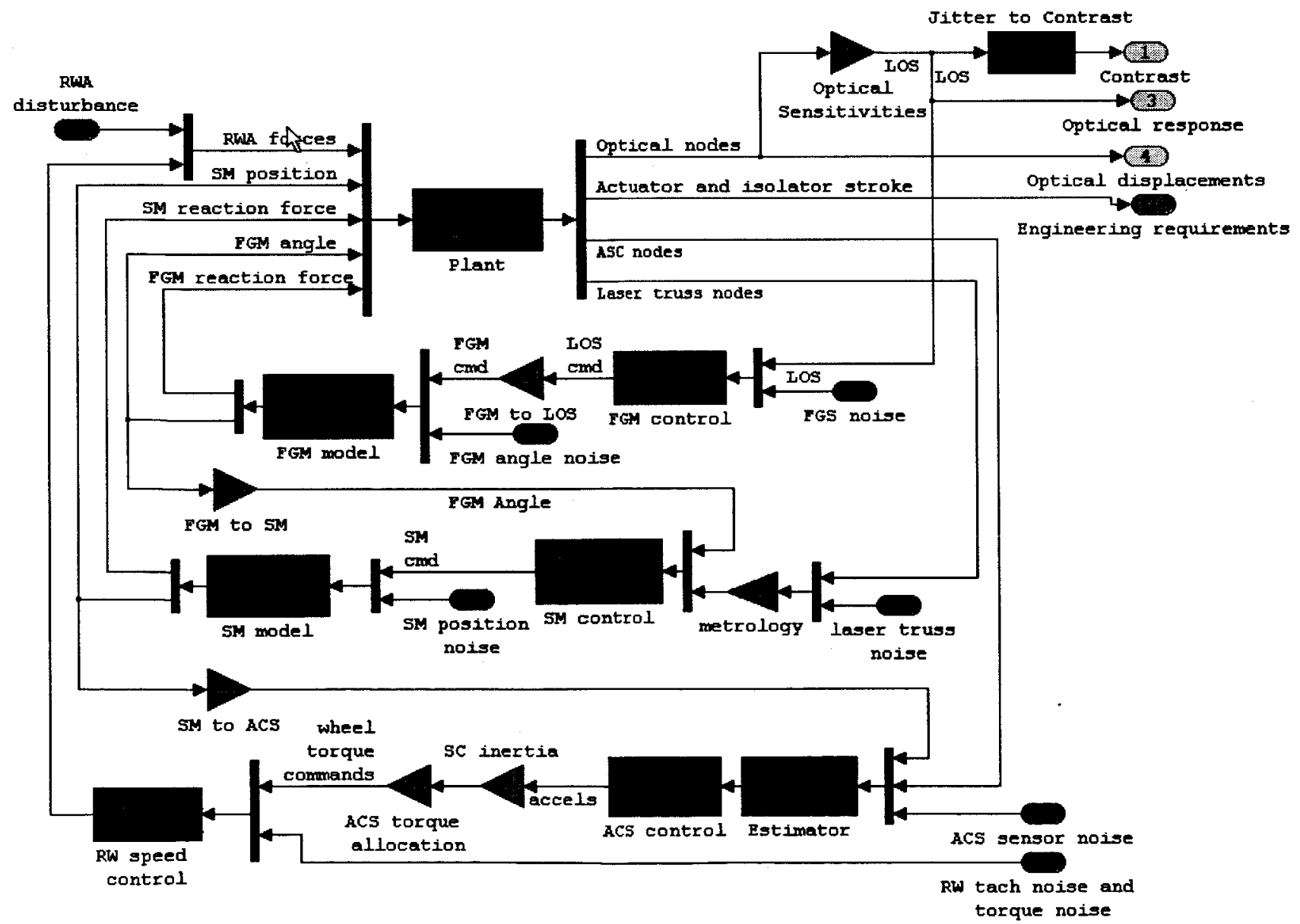

Figure 2-2 TPF-C integrated model configured for passive isolation. 


\section{INTEGRATED MODEL DESCRIPTION}

The integrated model is a fully coupled mathematical model of the dynamics of the FB1 design. The integrated model describes structural, optical, and contrast responses in the presence of dynamic disturbances. This section presents the components of the model.

\subsection{Integrated model overview}

The integrated model is shown in Figure 2-2. It consists of a structural plant model, optics, controllers, and additional component models.

\subsection{Structure model}

The structure model is a Finite Element model generated by JPL and GSFC. It is provided in the form of Normal Modes frequencies and modeshapes ${ }^{3}$. The Normal Modes model is converted to a state space model that maps physical inputs to nodal responses. Damping is modeled using modal damping on a component level, and allocated to the structural modes using a Strain Energy Fraction approach:

$$
\zeta_{i}=\sum_{j} F_{i j} \zeta_{j}
$$

where $\zeta_{j}$ is the modal damping for mode $i, F_{i j}$ is the element strain energy fraction in mode $i$ for component $\mathrm{j}$, and $\zeta_{j}$ is the modal damping ratio for component $j$. Damping values are based on a best estimate with a knockdown factor, and are tabulated in Table 3-1. The knockdown factors are lower when there is published data for a similar component, and higher in the absence of data.

The full order model has 7218 modes from 0 to $300 \mathrm{~Hz}$. Two reduced order models were used. A 1000 mode model was used for design, and a $\mathbf{2 0 0 0}$ mode model for analysis. Reduction was accomplished using tools in the DOCS Toolbox.

\subsection{Optics model}

The optics model is provided in the form of linear sensitivity matrices that map motions of the optical nodes of the FEM to optical responses': LOS, beam walk, and wave front error (aberration). The LOS optical model gives motion of the centroid on the occulting mask, which is converted to an angle on the sky using the system focal length. The beam walk sensitivities give the motions of the chief ray on each of the 21 optics, as a function of rigid-optic motion of each of the optics. The Structural Deformation and Optical Deformation sensitivities give the wave front error aberration, in Zemike amplitude normalized to unity RMS, from, respectively, rigid motion of the optical elements, and distortion of the Primary Mirror. Distortion of the downstream mirrors is budgeted but was not analyzed in the FBl cycle.

\subsection{Component models}

The integrated model also includes several components that are modeled separately in order to allow for parametric sweeps, and to model various sensing and computational processes.

\subsubsection{Secondary mirror and fine guidance mirror}

The SM and FGM are represented as position-controlled dynamic systems. For example, a piezoelectric steering mirror would transform a voltage command into an angular position change. The motion of the mirror would also generate a reaction force back into the structure. The devices also generate a constant position noise (and accompanying reaction force) as a result of electrical noise in the drive electronics.

\subsubsection{Metrology}

The laser truss metrology system measures the relative motion of the SM and PM by sending laser legs through retroreflectors on the SM and PM. The length change of the legs is transformed into relative motion. The measurement should be possible at very high frequency $(>1 \mathrm{kHz})$ so a static gain matrix is used to relate comer cube motions to SM-PM motion.

\subsubsection{Reaction wheel speed control}

The reaction wheel speed controllers are used to reduce the effects of spin-axis disturbances, most significantly torque noise. The wheel speed controllers use a tachometer to drive the wheels at a commanded spin rate, which reduces spin-axis disturbances below the controller bandwidth, at the cost of Table 3-1 Nominal component damping values.

\begin{tabular}{|l|l|l|l|}
\hline Component & $\begin{array}{l}\text { Best } \\
\text { estimate }\end{array}$ & $\begin{array}{l}\text { Knock } \\
\text { down }\end{array}$ & $\begin{array}{l}\text { Nominal } \\
\text { value }\end{array}$ \\
\hline Payload isolator & $5 \%$ & 2.0 & $2.5 \%$ \\
\hline RWA isolator & $5 \%$ & 1.2 & $4.17 \%$ \\
\hline Sunshield & $1 \%$ & 3 & $0.33 \%$ \\
\hline Bus & $0.5 \%$ & 5 & $0.1 \%$ \\
\hline OTA & $0.5 \%$ & 5 & $0.1 \%$ \\
\hline
\end{tabular}


Table 3-2 Actuator noise parameters.

\begin{tabular}{|l|l|l|}
\hline Noise source & Noise level & Bandwidth \\
\hline SM position error & $0.02 \mathrm{~nm}$ RMS & $0-50 \mathrm{~Hz}$ \\
& $0.02 \mathrm{nrad} \mathrm{RMS}$ & \\
\hline FGM position error & $10 \mathrm{masec}$ RMS & $0-250 \mathrm{~Hz}$ \\
\hline torque noise & $6 \mathrm{e}-4 \mathrm{~N}-\mathrm{m} / \mathrm{Hz}^{1 / 2}$ & $0.1-1 \mathrm{~Hz}$ \\
\hline
\end{tabular}

which contaminates the wheel command. The speed controller is implemented as an integral filter with a lead controller, with a bandwidth of $1 \mathrm{~Hz}$ and 60 degrees of lead. The tachometer is sampled at $100 \mathrm{~Hz}$.
Table 3-3 Sensor noise parameters.

\begin{tabular}{|c|c|c|}
\hline Noise source & RMS noise level & Bandwidth \\
\hline FGS & 0.005 masec $(\mathrm{X}$-axis $)$ & $500 \mathrm{~Hz}$ \\
\hline Laser $\mathbf{d f} / \mathrm{f}$ & $1 \mathrm{e}-9 \mathrm{~Hz} / \mathrm{Hz}$ & \multirow[t]{2}{*}{$1 \mathrm{kHz}$} \\
\hline Laser dL & $25 \mathrm{~nm}$ & \\
\hline Kalman filter & 0.120 masec & $2 \mathrm{~Hz}$ \\
\hline Star Tracker & $1.35 \mathrm{asec}$ & $2 \mathrm{~Hz}$ \\
\hline Gyro & $\begin{array}{l}6.8 \mathrm{e}-5 \mathrm{asec} / \mathrm{sec}^{3 / 2} \\
0.01 \mathrm{asec} / \mathrm{sec}^{1 / 2}\end{array}$ & $2 \mathrm{~Hz}$ \\
\hline PSAC & 0.135 asec & $2 \mathrm{~Hz}$ \\
\hline Tachometer & le-3 rad/sec & $100 \mathrm{~Hz}$ \\
\hline
\end{tabular}

\subsubsection{Estimator}

The estimator provides the measured attitude signal to the ACS control, with the measurement derived from the appropriate sensor for the control mode. Selection of the control mode also feeds the correct sensor noise source into the estimator. The estimator is realized as a static gain matrix.

\subsubsection{FGM, SM, and ACS controllers}

The controllers are represented as state space systems that map sensor signals to control signals. All controllers include Pade approximations of the sample delay.

\subsection{Disturbance Models}

The disturbances acting on TPF-C are generally random processes that can be modeled as colored noise. The exceptions are the reaction wheel spin-rate-dependent forces, which are modeled using discrete tonal forces.

\subsubsection{Reaction wheel spin-rate-dependent forces}

The RW imbalance forces are modeled as a sum of sinusoidal disturbances at harmonics of the wheel speed:

$$
d(t)=\sum_{i=1}^{N_{h}} C_{i} f_{\mathrm{rw}}^{2} \sin \left(2 \pi h_{i} f_{\mathrm{rw}} t+\phi_{i}\right)
$$

where $C_{i}$ is the amplitude coefficient, $f_{\mathrm{rw}}$ is the reaction wheel speed, $h_{i}$ is the $\mathrm{i}^{\text {th }}$ harmonic, and $\phi_{i}$ is the phase associated with the $i^{\text {th }}$ harmonic.

\subsubsection{Sensor noise}

The various sensor noises are modeled as sampled continuous time white noise. The sampling process is approximated by a 1-pole continuous time low pass filter. The filter matches the DC magnitude of the noise, so it over-predicts the
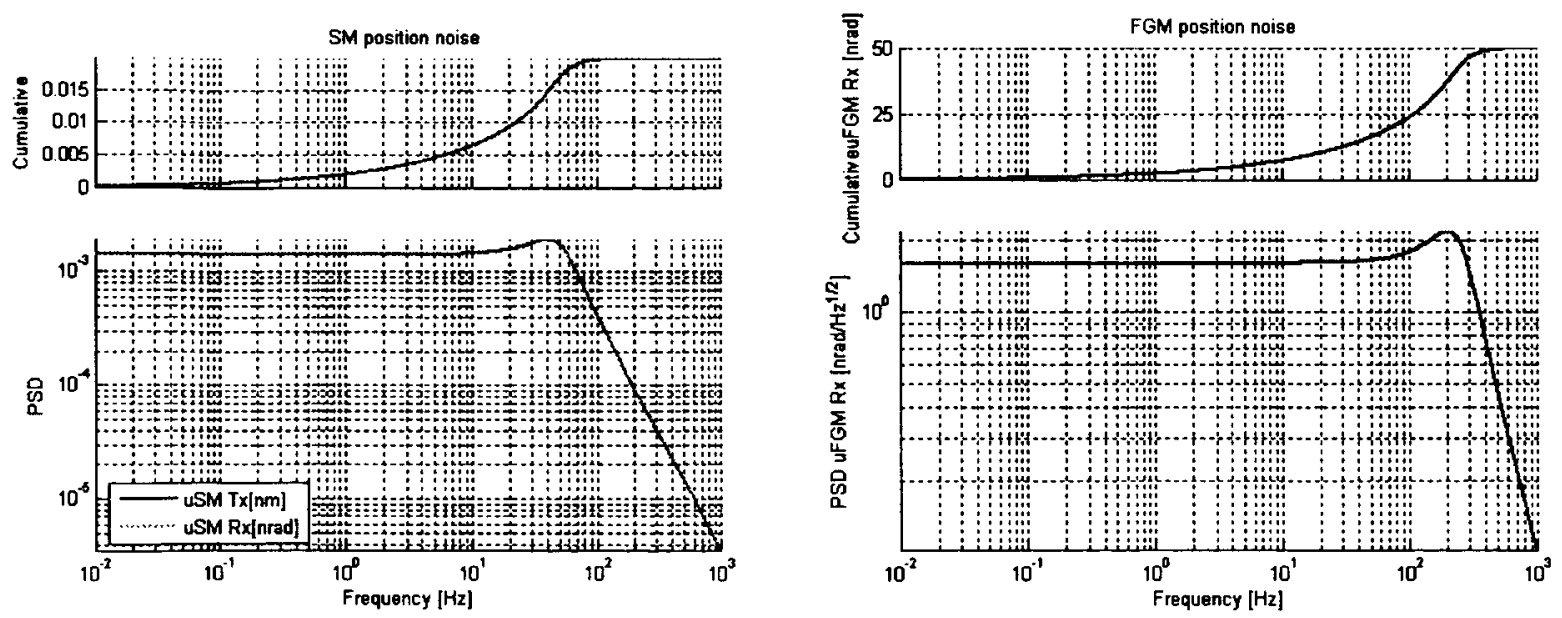

Figure 3-1 Left, RMS SM position noise, translation (solid) and rotation (dotted). Right, FGM position error. 
RMS noise by $25 \%$. Sensor noises are assumed on the Fine Guidance Sensor, laser truss for SM position measurement (due to measurement error and frequency error), Star Tracker and gyro, PSAC, and RW tachometer. In coarse mode, the Star Tracker and gyro are combined using a Kalman Filter that lowers the noise level. The sensor noise values are given in Table 3-3.

\subsubsection{Actuator noise}

The feedback actuators are all subject to various non-idealities that inject noise into the plant. The reaction wheels have a number of characteristics that create spin-axis forces. The TPF-C Minimum Mission analysis showed that one of the strongest drivers was torque noise.

The two mirrors that are actively controlled are also subject to position noise. The amplifiers that provide the high voltage signals are subject to electronic noise, that feeds through the actuators to generate a position noise. The noise bandwidth is set by the resonance of the device. Figure 3-1 plots the assumed position noise spectra for (left) the secondary mirror actuators, and (right) the Fine Guidance Mirror. The actuator noise values are tabulated in Table 3-2.

\subsection{Analysis tools}

The structural model is developed in NASTRAN. The optical sensitivity matrices are created in MACOS. The model is integrated and analyzed in MATLAB® using the DOCS® Toolbox.

\section{PERFORMANCE MODELING}

\subsection{Structural and optical responses}

The RW analysis is conducted using a discrete wheel speed analysis. The disturbances are represented in the frequency domain and applied to the frequency response function (FRF) of the structure to predict the complex-valued optical performance:

$$
\begin{aligned}
& d\left(j \omega_{i}\right)=C_{i} f_{\mathrm{rW}}^{2} \delta\left(j \omega_{i}\right) e^{j \phi_{i}} \\
& z(j \omega)=\frac{1}{\sqrt{2}} \sum_{i=1}^{N_{h}} G_{z d}\left(j \omega_{i}\right) d\left(j \omega_{i}\right)
\end{aligned}
$$

where $\omega_{i}=2 \pi y_{\mathrm{rw}} h_{i}, \delta(\cdot)$ is the dirac-delta function, $N_{h}$ is the total number of harmonics used, and $G_{z d}$ is the frequency response function from wheel disturbance $d$ to optical performance $z$. The sensor noise analysis is conducted using a continuous PSD analysis:

$$
\begin{aligned}
& S_{z z}(j \omega)=G_{z w}^{H}(j \omega) S_{z z}(j \omega) G_{z w}(j \omega) \\
& \Sigma_{z z}=\frac{1}{2 \pi} \int S_{z z}(j \omega) \partial \omega
\end{aligned}
$$

where $S_{z z}$ is the disturbance PSD, $G_{z w}$ is the frequency response function from the disturbance to the optical performance, and $\Sigma_{z z}$ is the covariance of the optical response.

\subsection{Model uncertainty}

The predicted structural and optical responses were multiplied by a frequency dependent Model Uncertainty Factor that reflects the potential model error in the different components of the system. The low frequency $(0-20 \mathrm{~Hz})$ value was 3 , above $40 \mathrm{~Hz}$ the value was 10 , with a linear transition $20-40 \mathrm{~Hz}$. The MUF levels were chosen on a component level, based on the maturity of the component (high for the RWA isolator, low for the telescope) and RSSed.

\subsection{Contrast computations}

The contrast degradation from the predicted optical response is generated using the following equations, taken from the TPF-C error budget.

\subsubsection{LOS}

The contrast degradation produced by image jitter, $\sigma$, and offset $\Omega$, is given by

$$
C_{L O S}=c_{1} \sigma^{4} \Omega^{4}+c_{2} \sigma^{6} \Omega^{2}+c_{3} \sigma^{2} \Omega^{6}+c_{4} \sigma^{8}+c_{5} \Omega^{8}
$$

This equation was developed using Monte Carlo simulation and a curve fit. 


\subsubsection{Beam walk}

The beam walk contrast degradation for a particular optic is given by

$$
C_{B W, i}=A_{i} \frac{\sigma_{x}^{2}+\sigma_{y}^{2}+\sigma_{z}^{2}}{\Delta \sigma_{x}^{2}}
$$

where the $A_{i}$ constant is derived from an area integral of surface roughness, $\sigma_{\mathrm{x}}, \sigma_{\mathrm{y}}, \sigma_{\mathrm{z}}$ are the RMS motions across the optic in the $\mathrm{X}, \mathrm{Y}$, and $\mathrm{Z}$ axes respectively, and $\Delta \sigma_{\mathrm{x}}$ is a normalizing wavelength. The beam walk contrasts are computed individually for each of the 21 optics, and summed to produce a total contrast.

\subsubsection{Aberrations}

Contrast due to Structural Deformation and Optical Deformation aberrations are both computed using a polynomial fit:

$$
C_{j}=10^{a_{j}}\left(\frac{Z r m s_{j}}{\lambda \cdot 10^{10}}\right)^{P_{j}}
$$

where $Z r m s_{j}$ is the RMS amplitude of Zernike mode $j$ in Angstroms, $\lambda$ is the wavelength in meters, and $a_{j}$ and $P_{j}$ are the polynomial coefficients.

\subsubsection{Mask errors}

The mask error contrast is a polynomial in the Zernike amplitude, given by:

$$
C_{M E, j}=\left(\frac{Z r m s_{j}}{\lambda \cdot 10^{10}} 10^{a_{j}} 4^{p_{j}} \Delta M\right)^{2}
$$

where $Z r m s_{j}$ is the RMS amplitude of Zernike mode $j$ in Angstroms, $\lambda$ is the wavelength in meters, $a_{j}$ and $p_{j}$ are the polynomial coefficients, and $\Delta \mathrm{M}=1 \mathrm{e}-3$. The mask error contrast due to $L O S$ can be computed using the same equation, using a scale factor, 1.9582 , from radians to Zernike RMS amplitude in tip/tilt.

\section{JTTTER ANALYSIS}

The following sections present the predicted contrast due to jitter for the FB1 design.

\subsection{Optical element jitter}

The rigid body pointing (RBP) versus wheel speed of the closed loop system is shown in Figure 5-1, for the body X (solid), body $\mathrm{Y}$ (dashed), and body $\mathrm{Z}$ (dotted). The maximum response above the minimum wheel speed of $3 \mathrm{RPS}$ is 0.7 masec for the X-axis rotation. The peak RBP allocation depends on the authority of the secondary mirror control loop, and ranges from 4 masec at low frequency (where the SM is active) to 0.04 masec when the SM is effectively uncontrolled.

The peak RMS motions of the optical elements under the RW disturbance are plotted in Figure 5-2. The upper left plot shows the RMS transiations in nanometers, while the upper right plot shows the RMS rotations in nano-radians. The legend calls out the peak response frequency. The motions are defined relative to the PM, so the PM response is 0 . The requirements on the SM are $5 \mathrm{~nm}$ or lower in 3 axes of translation, and $5 \mathrm{nrad}$ or lower in 3 axes of rotation. The responses are significantly higher, $57.6 \mathrm{~nm}$ at the $1^{\text {st }} \mathrm{Y}$ tower bending mode, and $71.5 \mathrm{~nm}$ at the $1^{\text {st }} \mathrm{X}$ tower bending mode. The rotation ranges from 4.8 to 7.1 nrad depending on the axis. The downstream optics are below the requirement of 10 nrad per axis in rotation, and $100 \mathrm{~nm}$ per axis in translation. The net effect will depend on the resulting contrast, which is driven primarily by the beam walk term.

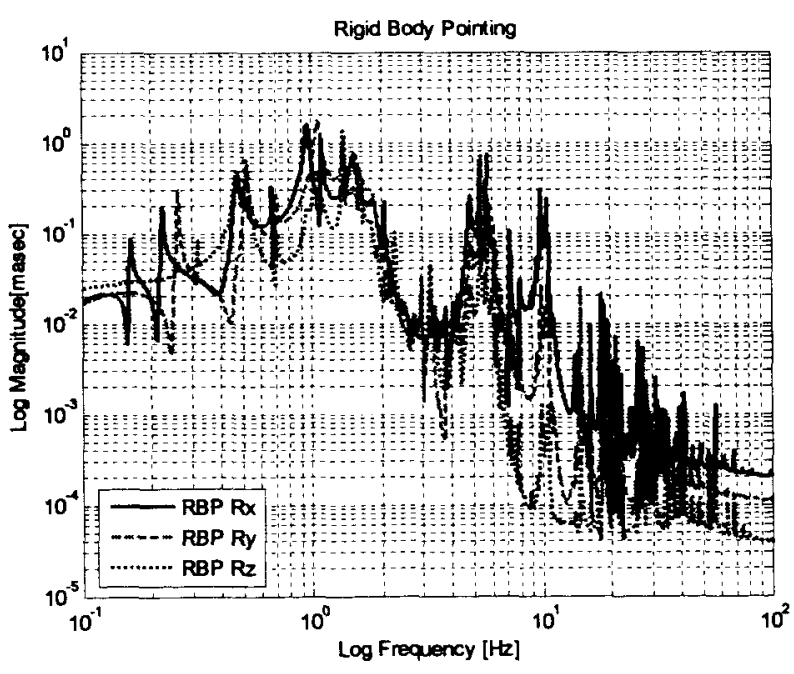

Figure 5-1 Rigid body pointing. 

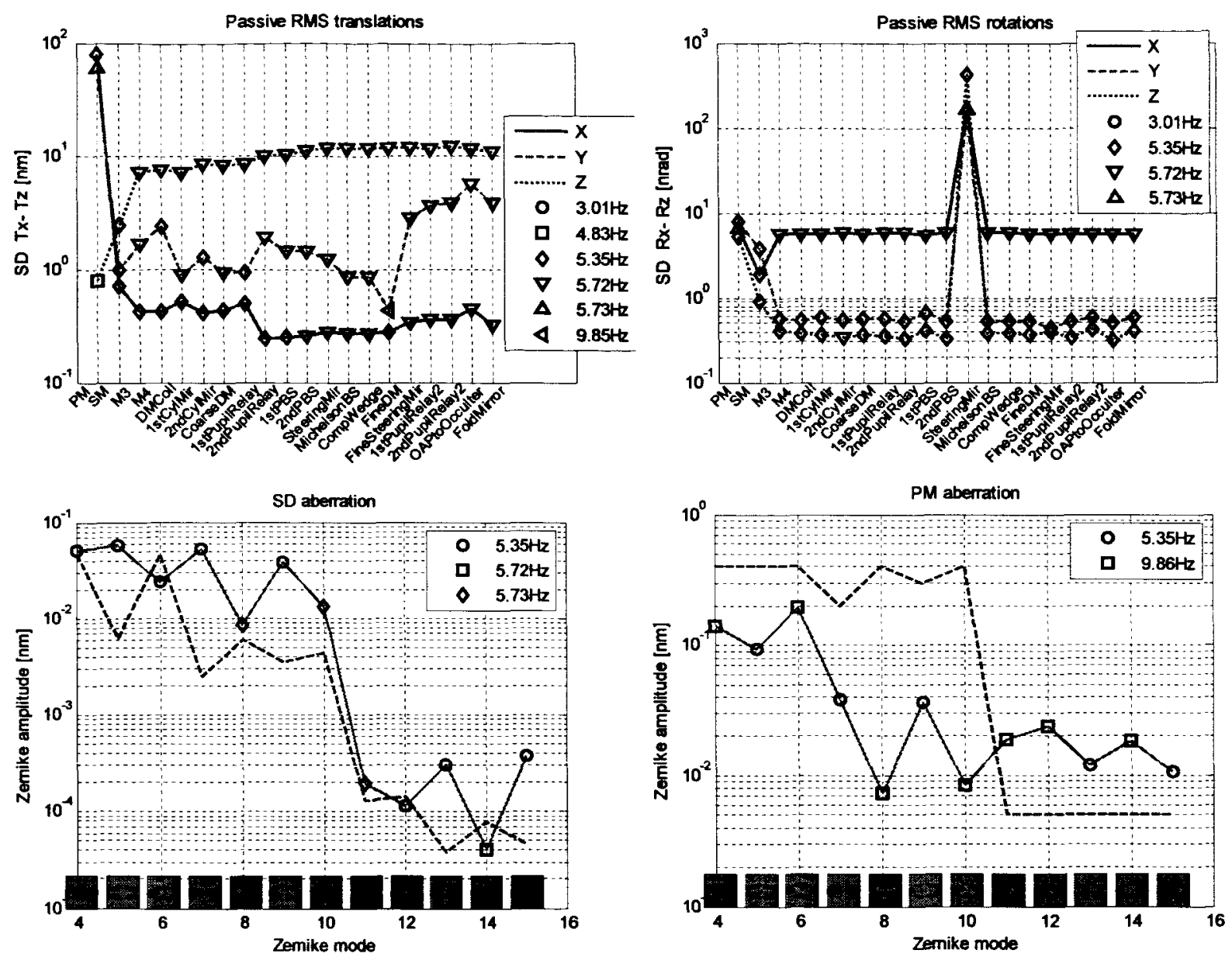

Figure 5-2 Jitter predictions under RW disturbance. Upper left, RMS translations of the optics. Upper right, RMS rotations. Lower left, RMS SD Zernike amplitudes. Lower right, RMS PM Zernike amplitudes.

The lower two plots show the Zernike amplitudes from structural deformation (left) and PM deformation (right). The dashed lines plot the requirements. The structural deformation aberrations are above the requirement by about a decade. The primary mirror deformation exceeds the requirement at the higher Zernike modes. The exceedances are not as problematic as the SM motion since the aberration contributions to contrast are about two orders of magnitude below the beam walk.

\subsection{Jitter induced contrast}

The peak jitter induced contrast is summarized in Table 5-1. The columns indicate the disturbance source, and the rows indicate the contrast contribution. The last column gives the requirement for each contribution, and for the total contrast.

The largest contrast contributions are the beam walk, and the primary mirror deformation. The largest contributor by disturbance source is the RW imbalance. Next largest is the RW torque noise, followed by the secondary mirror position error. The next section looks at design alternatives that would reduce reaction wheel imbalance induced contrast.

\subsection{Jitter sensitivities}

The baseline passive PCS design was perturbed in order to assess the performance sensitivity. The parameters were chosen based on a combination of anticipated influence on the cost, and ease of modification. Four parameters were chosen: 
Table 5-1 FB1 jitter induced contrast.

\begin{tabular}{|l|c|l|l|l|l|l|l|l|l|l|}
\hline & $\begin{array}{l}\text { PSAC } \\
\text { noise }\end{array}$ & $\begin{array}{l}\text { FGS } \\
\text { noise }\end{array}$ & $\begin{array}{l}\text { Tach } \\
\text { noise }\end{array}$ & $\begin{array}{l}\text { FGM } \\
\text { posn }\end{array}$ & $\begin{array}{l}\text { SM } \\
\text { posn }\end{array}$ & $\begin{array}{l}\text { Laser } \\
\text { truss }\end{array}$ & $\begin{array}{l}\text { RW } \\
\text { torque } \\
\text { noise }\end{array}$ & $\begin{array}{l}\text { RW } \\
\text { imb. }\end{array}$ & Total & Req \\
\hline Beam walk & 0.00 & $9.5 \mathrm{e}-17$ & $5.3 \mathrm{e}-14$ & $2.1 \mathrm{e}-13$ & $3.0 \mathrm{e}-14$ & $2.9 \mathrm{e}-15$ & $1.0 \mathrm{e}-12$ & $7.7 \mathrm{e}-12$ & $9.0 \mathrm{e}-12$ & $1.9 \mathrm{e}-12$ \\
\hline LOS & $1.2 \mathrm{e}-20$ & $1.2 \mathrm{e}-20$ & $1.2 \mathrm{e}-20$ & $2.2 \mathrm{e}-15$ & $7.5 \mathrm{e}-18$ & $2.4 \mathrm{e}-20$ & $1.2 \mathrm{e}-20$ & $8.1 \mathrm{e}-17$ & $2.3 \mathrm{e}-15$ & $9.0 \mathrm{e}-14$ \\
\hline LOS maskerr & $6.4 \mathrm{e}-14$ & $6.4 \mathrm{e}-14$ & $6.4 \mathrm{e}-14$ & $1.8 \mathrm{e}-13$ & $8 . \mathrm{e}-14$ & $1.3 \mathrm{e}-13$ & $6.4 \mathrm{e}-14$ & $9.7 \mathrm{e}-14$ & $7.4 \mathrm{e}-13$ & $5.5 \mathrm{e}-13$ \\
\hline SD aber & 0.00 & $9.2 \mathrm{e}-25$ & $3.6 \mathrm{e}-19$ & $5.2 \mathrm{e}-22$ & $2.0 \mathrm{e}-18$ & $5.0 \mathrm{e}-19$ & $5.0 \mathrm{e}-19$ & $1.6 \mathrm{e}-16$ & $1.6 \mathrm{e}-16$ & $2.8 \mathrm{e}-17$ \\
\hline SD maskerr & 0.00 & $1.2 \mathrm{e}-24$ & $2.4 \mathrm{e}-19$ & $1.1 \mathrm{e}-20$ & $5.0 \mathrm{e}-19$ & $1.7 \mathrm{e}-19$ & $2.2 \mathrm{e}-19$ & $7.9 \mathrm{e}-17$ & $8.0 \mathrm{e}-17$ & $1.7 \mathrm{e}-17$ \\
\hline PM deform & 0.00 & $2.9 \mathrm{e}-19$ & $2.0 \mathrm{e}-17$ & $2.6 \mathrm{e}-16$ & $1.7 \mathrm{e}-12$ & $4.9 \mathrm{e}-18$ & $6.9 \mathrm{e}-17$ & $1.8 \mathrm{e}-12$ & $3.5 \mathrm{e}-12$ & $8.6 \mathrm{e}-13$ \\
\hline PM maskerr & 0.00 & $1.1 \mathrm{e}-22$ & $2.1 \mathrm{e}-20$ & $8.3 \mathrm{e}-20$ & $3.0 \mathrm{e}-16$ & $1.8 \mathrm{e}-21$ & $7.8 \mathrm{e}-20$ & $4.9 \mathrm{e}-16$ & $7.8 \mathrm{e}-16$ & $5.2 \mathrm{e}-15$ \\
\hline Total & $6.4 \mathrm{e}-14$ & $6.4 \mathrm{e}-14$ & $1.2 \mathrm{e}-13$ & $3.9 \mathrm{e}-13$ & $1.8 \mathrm{e}-12$ & $1.3 \mathrm{e} 13$ & $1.1 \mathrm{e}-12$ & $8.0 \mathrm{e}-12$ & $1.2 \mathrm{e}-11$ & $3.4 \mathrm{e}-12$ \\
\hline
\end{tabular}

1. FGM reactuation: an equivalent inertia can be driven $180^{\circ}$ out of phase with the FGM, partially canceling the reaction force on the structure. This is commonly required for high performance optical systems, since the FGM acts as a high frequency disturbance source on the quiet side of the isolator

2. SM reactuation: the secondary mirror assembly (SMA) for the baseline design is significantly over its mass budget, necessitating a redesign, so the effect on dynamic performance is an important question

3. $20 \mathrm{~Hz}$ SM bandwidth : this will reduce the beam walk contribution from the tower modes at $5 \mathrm{~Hz}$. In order to maintain loop stability, the SM must be decoupled from the tower modes using SM reactuation.

4. $2 \%$ damping in the tower $1^{\text {st }}$ and $2^{\text {nd }}$ modes at 5 and $10 \mathrm{~Hz}$ : a passive damping treatment can reduce the dynamic amplification in these regions.

The sensitivity study looked only at the RW imbalance disturbance, since this is the largest contrast contributor. The sensitivity study was performed on a reduced order $(250$ mode $)$ model, so the results are slightly different for the nominal.

The contrast predictions for the nominal design and four perturbed designs is given in Table 6-1. The columns give the design case, and rows give the contrast contribution. The last column gives the contrast requirement. The FGM reactuation does not change the contrast prediction at all. This is an indication that the FGM inertia is so low that the reaction forces are negligible. The next perturbation, SM reactuation, actually increases several of the contrast contributions. This results from a shift in a critical mode frequency, that causes it to couple more strongly to an adjacent mode and therefore to increase in magnitude. The conclusion is that lightweighting the SMA, while desirable from the standpoint of increasing the tower mode frequencies, will not change the induced vibration significantly, because of the low bandwidth of the SM loop. This conclusion could change if the SM loop bandwidth were increased. The $20 \mathrm{~Hz} \mathrm{SM}$ loop does reduce the beam walk contrast. However, the PM deformation contribution is unaffected (it increases due to the SM reactuation), so the total contrast is not reduced enough to meet requirements. The tower damping case significantly reduces the contrast. Under this scenario, the total contrast would drop to $3.85 \mathrm{e}-12$.

\section{CONCLUSIONS AND FUTURE WORK}

The paper has presented a snapshot of the FB1 passive jitter design activities. The results show that the current system does not meet requirements, but a number of promising design modifications might lead to a feasible design. They include

- Passive damping treatments. In addition to reducing the nominal contrast, this would represent a significant risk buydown, by augmenting the highly uncertain inherent structural damping with a linear damping mechanism that could be well characterized prior to flight.

- Noise filtering. The large contribution from the SM position noise could be reduced by filtering the drive amplifier output above the SM control bandwidth.

In addition, a number of additional sensitivity studies are planned to examine: 
- damping assumptions

- control system optimization (of the loop shaping designs as well as a modern optimal control system)

- parameter uncertainty, including variability in critical structural stiffnesses

These sensitivity studies will address system optimization for cost and risk.

\section{ACKNOWLEDGEMENTS}

The authors would like to thank Andy Kissil at JPL and Sandra Irish at GSFC for support with the structural modeling, Philip Dumont at JPL for assistance with the optical modeling, and Stuart Shaklin at JPL with support for the error budgeting.

\section{REFERENCE}

1. M. Levine, M. White, et. al., Terrestrial Planet Finder Coronagraph - Minimum Mission Baseline Design and Analysis Report, JPL Document 28535, 2004.

2. T. Hyde, K. Ha, J. Johnston, J. Howard, and G. Mosier, "Integrated modeling activities for the James Webb Space Telescope: optical jitter analysis", SPIE Astronomical Telescopes and Instrumentation, Glasgow, UK, 2004.

3. A. Kissil, "Structural Modeling for the Terrestrial Planet Finder Mission", SPIE Optical Science and Technology, Denver, Colorado, 2004.

4. P. Davis, D. Cunningham, and J. Harrell, “Advanced $1.5 \mathrm{~Hz}$ Passive Viscous Isolation System", $35^{\text {th }}$ AIAA SDM Conference, Hilton Head, South Carolina, 1994.

5. S. Basinger and D. Redding, "Terrestrial Planet Finder Coronagraph Optical Modeling", SPIE Optical Science and Technology, Denver, Colorado, 2004.

6. S. B. Shaklin, L. F. Marchen, J. J. Green, "The Terrestrial Planet Finder Coronagraph error budget", SPIE Optics and Photonics, San Diego, 2005.

Table 6-1 RW jitter sensitivities.

\begin{tabular}{|l|l|l|l|l|l|l|}
\hline \hline Design case & Nominal & Reactuated FGM & Reactuated SM & 20 Hz SM & $2 \%$ Twr Damping & Req \\
\hline \hline Beam walk & $7.77 \mathrm{e}-12$ & $7.77 \mathrm{e}-12$ & $8.50 \mathrm{e}-12$ & $5.04 \mathrm{e}-14$ & $3.66 \mathrm{e}-13$ & $1.9 \mathrm{e}-12$ \\
\hline LOS & $7.69 \mathrm{e}-17$ & $7.69 \mathrm{e}-17$ & $1.10 \mathrm{e}-16$ & $1.23 \mathrm{e}-20$ & $1.01 \mathrm{e}-19$ & $9.0 \mathrm{e}-14$ \\
\hline LOS maskerr & $9.66 \mathrm{e}-14$ & $9.66 \mathrm{e}-14$ & $1.01 \mathrm{e}-13$ & $6.05 \mathrm{e}-14$ & $6.27 \mathrm{e}-14$ & $5.5 \mathrm{e}-13$ \\
\hline SD aber & $1.62 \mathrm{e}-16$ & $1.62 \mathrm{e}-16$ & $1.86 \mathrm{e}-16$ & $8.08 \mathrm{e}-18$ & $3.62 \mathrm{e}-18$ & $2.8 \mathrm{e}-17$ \\
\hline SD maskerr & $7.99 \mathrm{e}-17$ & $7.99 \mathrm{e}-17$ & $8.74 \mathrm{e}-17$ & $1.13 \mathrm{e}-17$ & $2.70 \mathrm{e}-18$ & $1.7 \mathrm{e}-17$ \\
\hline PM deform & $1.77 \mathrm{e}-12$ & $1.77 \mathrm{e}-12$ & $2.01 \mathrm{e}-12$ & $2.01 \mathrm{e}-12$ & $2.07 \mathrm{e}-13$ & $8.5 \mathrm{e}-13$ \\
\hline PM maskerr & $4.82 \mathrm{e}-16$ & $4.82 \mathrm{e}-16$ & $5.47 \mathrm{e}-16$ & $5.47 \mathrm{e}-16$ & $9.64 \mathrm{e}-17$ & $5.2 \mathrm{e}-15$ \\
\hline Total & $9.63 \mathrm{e}-12$ & $9.63 \mathrm{e}-12$ & $1.06 \mathrm{e}-11$ & $2.12 \mathrm{e}-12$ & $6.36 \mathrm{e}-13$ & $3.4 \mathrm{e}-12$ \\
\hline
\end{tabular}

